\title{
Usefulness of intraoperative bronchoscopy during surgical repair of a congenital cardiac anomaly with possible airway obstruction -three cases report-
}

\author{
JongEun Oh, Jung-won Kim, Won-Jung Shin, Mijeung Gwak, and \\ Pyung Hwan Park
}

Department of Anesthesiology and Pain Medicine, Asan Medical Center, Seoul, Korea

Compression of the airway is relatively common in pediatric patients, although it is often an unrecognized complication of congenital cardiac and aortic arch anomalies. Aortopexy has been established as a surgical treatment for tracheobronchial obstruction associated with vascular anomaly, aortic arch anomaly, esophageal atresia, and tracheoesophageal fistula. The tissue-to-tissue arch repair technique could result in severe airway complication such as compression of the left main bronchus which was not a problem before the correction. We report three cases of corrective open heart surgery monitored by intraoperative bronchoscopy performed during prebypass, and performed immediately before weaning from bypass, to evaluate tracheobronchial obstruction caused by congenital, complex cardiac anomalies in the operating room.

Key Words: Airway obstruction, Aortic coarctation, Broncoscope, Coarctation of the aorta, Congenial heart disease.

Airway compression is relatively common in pediatric patients, although it is often an unrecognized complication of congenital cardiac and aortic arch anomalies. Aortopexy has become established as a successful surgical treatment for tracheo-

Corresponding author: Mijeung Gwak, M.D., Ph.D.

Department of Anesthesiology and Pain Medicine, Asan Medical Center, 88, Olympic-ro 43-gil, Songpa-gu, Seoul 05505, Korea

Tel: 82-2-3010-3868, Fax: 82-2-3010-6790

E-mail: mjgwak@amc.seoul.kr

Received: December 29, 2014.

Revised: 1st, May 11, 2015; 2nd, June 11, 2015; 3rd, June 13, 2015; 4th, June 18, 2015.

Accepted: June 26, 2015.

Korean J Anesthesiol 2016 February 69(1): 71-75

http://dx.doi.org/10.4097/kjae.2016.69.1.71 bronchial obstruction associated with vascular anomaly, aortic arch anomaly, esophageal atresia, and tracheoesophageal fistula as the tissue-to-tissue arch repair technique can result in severe airway complications, such as compression of the left main bronchus, and which were not a problem before the correction [1]. We report three cases of corrective open-heart surgery monitored by intraoperative bronchoscopy performed during prebypass and performed immediately before weaning from bypass, in order to evaluate tracheobronchial obstruction caused by congenital, complex, cardiac anomalies.

\section{Case Reports}

\section{Case 1}

A 4-year-old female was diagnosed with an ascending aortic

(c) This is an open-access article distributed under the terms of the Creative Commons Attribution Non-Commercial License (http://creativecommons.org/ licenses/by-nc/4.0/), which permits unrestricted non-commercial use, distribution, and reproduction in any medium, provided the original work is properly cited. 
aneurysm. She did not have dyspnea or cyanosis except for a murmur detected on physical examination. Preoperative 2D echocardiography showed that the right pulmonary artery was compressed by the dilated ascending aorta. A preoperative heart computed tomography (CT) scan revealed very severe focal stenosis in the right pulmonary artery antero-posteriorly compressed by the dilated ascending aorta. Consolidations involving the left lung field with upper lung predominance as well as the right upper lung field were also observed. The midportion of the left main bronchus was also severely compressed by the dilated ascending aorta (Fig. 1A), as seen by bronchoscopy in the operating room. The patient underwent aortic graft interposition as well as aortopexy. At the end of the surgery, we confirmed tracheobronchial decompression using intraoperative bronchoscopy (OLYMPUS VISERA OTV-S7 digital processor, OLYMPUS VISERA CLV-S40, [3.2 mm], OLYMPUS Medical Systems Corporation, Shinjuku-ku Tokyo, Japan) (Fig. 2). A postoperative heart CT scan showed improvement of the right pulmonary artery compression and normalization of the previ- ously severe narrowing of the mid-portion of the left main bronchus (Fig. 1B).

\section{Case 2}

A 10-day-old female was diagnosed with absent pulmonary valve syndrome with pulmonary regurgitation. She suffered respiratory distress during the immediate postnatal period and which required endotracheal intubation and mechanical ventilation. Preoperative 2D echocardiography showed the absence of a pulmonary valve and severe pulmonary regurgitation. It also revealed right ventricular hypertrophy, a subarterial ventricular septal defect (VSD), bilateral pulmonary artery enlargement, and patent ductus arteriosus (PDA). A heart CT scan showed compression of the left posterolateral portion of the lower trachea caused by the dilated aorta. The left main bronchus between the right pulmonary artery and the descending thoracic aorta was most severely compressed. The patient underwent anterior aortopexy of the ascending aorta as well as PDA division.
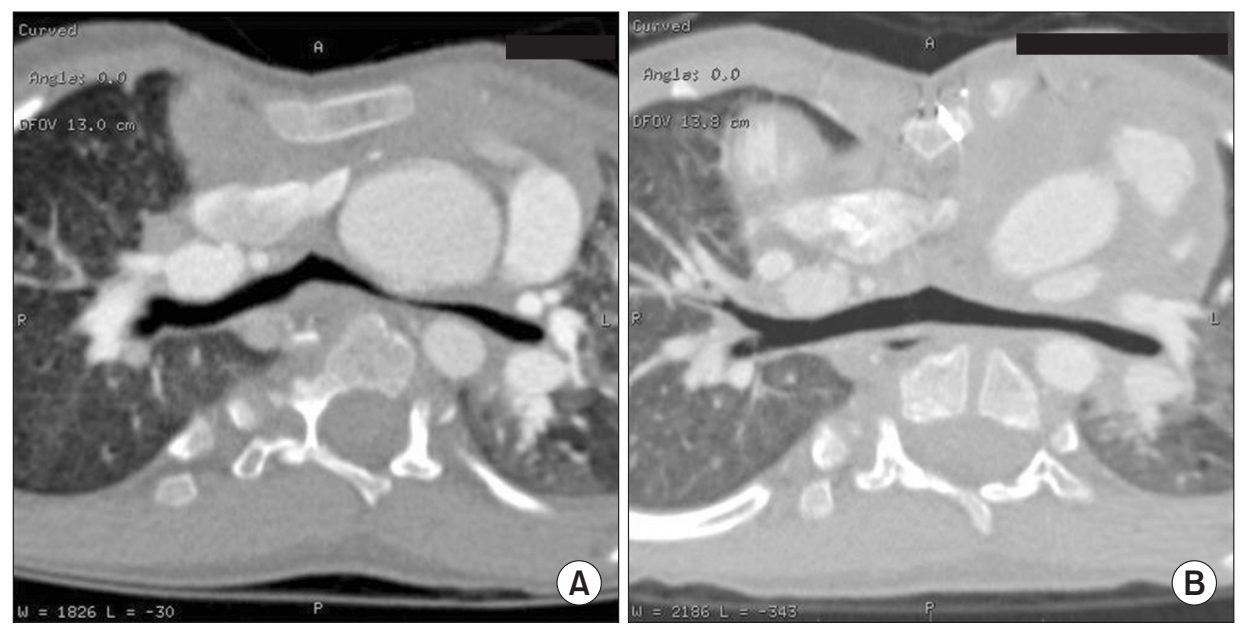

Fig. 1. The mid-portion of the left main bronchus between the dilated ascending aorta and the thoracic vertebra was severely compressed (A). The post-aortopexy heart CT scan showed less narrowing at the mid-portion of the left main bronchus (B).
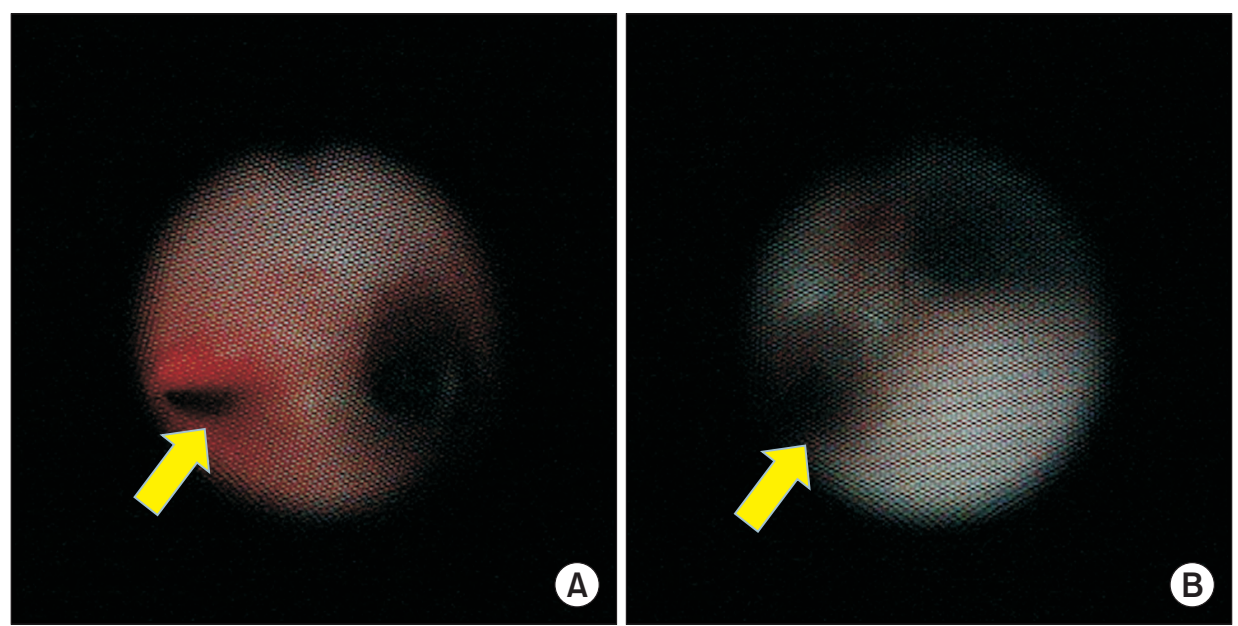

Fig. 2. Bronchoscopic view of the severe narrowing of the left main bronchus (A). At the end of the surgery, intraoperative bronchoscopy confirmed improvement of the previous bronchial compression (B). Yellow arrows indicate the opening of the left main bronchus. 
This procedure included the approximation of the pulmonary artery to the left lateral aspect of the ascending aorta. At the end of the surgical procedure, we confirmed tracheobronchial decompression by intraoperative bronchoscopy $(2.1 \mathrm{~mm})$. The patient had been successfully extubated by postoperative day 4 and had no further respiratory difficulty.

\section{Case 3}

A 7-day-old female infant was admitted due to her need for surgery for co-arctation of the aorta (CoA) as she had a double-outlet right ventricle (DORV) with sub-pulmonic VSD, otherwise referred to as Taussig-Bing anomaly. She was diagnosed during the antenatal period. She had tachypnea and mild peripheral cyanosis. Physical examination revealed an imperforated anus. Preoperative 2D echocardiography revealed DORV, a large VSD, and isthmic hypoplasia of the aorta. A preoperative heart CT scan also showed CoA with hypoplasia of the distal transverse arch, DORV, and multiple VSDs. It showed cardio- megaly with an enlarged right cardiac chamber, although there was no evidence of central airway narrowing. The patient had underwent arch reconstruction (extended end-to-end anastomosis) of severe transverse and isthmic tubular hypoplasia under selective cerebral perfusion as well as pulmonary artery banding with surgical tape. In the operating room after the procedure we found focal narrowing of the left main bronchus using bronchoscopy $(2.1 \mathrm{~mm})$ and notified the surgeon of this finding. However, the surgeon suggested that the patient's condition would improve after treatment using ventilator support. Therefore, the patient was delivered to the pediatric intensive care unit. However, postoperative extubation failed and her respiratory difficulty persisted. A postoperative heart CT scan showed newly developed, severe focal narrowing at the midportion of the left main bronchus between the right pulmonary artery and the midline descending thoracic aorta after arch repair and pulmonary artery banding (Fig. 3A). Using intraoperative bronchoscopy, we found that the left main bronchus was severely collapsed between the right pulmonary artery and the
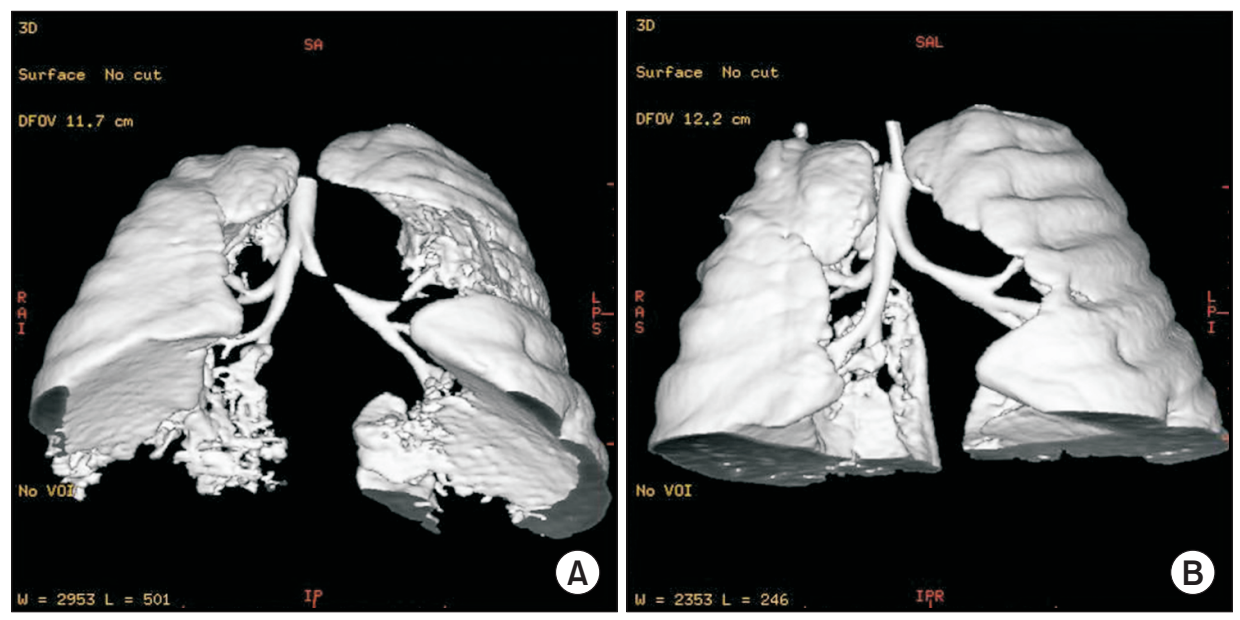

Fig. 3. Heart CT scan image obtained following the first surgery showed severe focal narrowing at the mid-portion of the left main bronchus (A). Following reoperation, improvement of the narrowing of the left main bronchus was revealed on the postoperative heart CT scan (B).
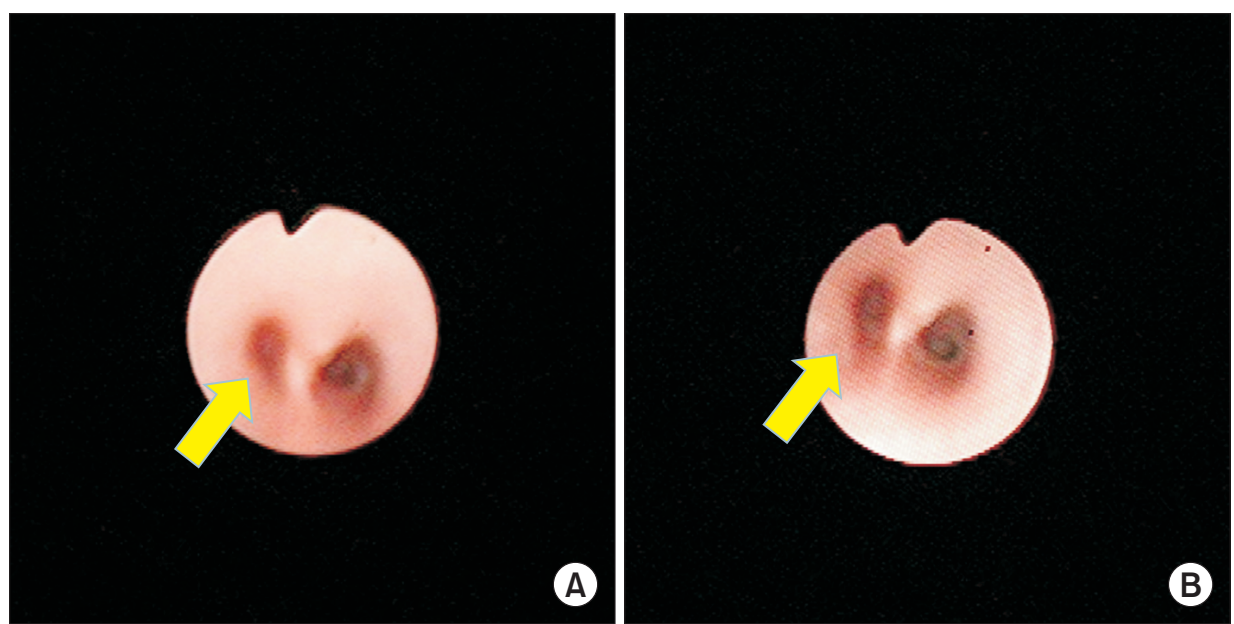

Fig. 4. The left main bronchus was severely collapsed between the right pulmonary artery and the descending aorta, as seen in the intraoperative bronchoscopic view (A). After pulmonary arteriopexy anterior to the ascending aorta, the collapsed left main bronchus was improved and confirmed by intraoperative bronchoscopy (B). The yellow arrows indicate the opening of the left main bronchus. 
descending aorta (Fig. 4A). On postoperative day 21, the patient underwent pulmonary arteriopexy anterior to the ascending aorta, whereupon the collapse of the left main bronchus was improved and confirmed by intraoperative bronchoscopy (Fig. $4 \mathrm{~B})$. We also observed improvement of the narrowing of the left main bronchus on the postoperative heart CT scan (Fig. 3B).

\section{Discussion}

The airway pathology caused by congenital heart disease in infants and children would be secondary to intrinsic or extrinsic causes or a combination of both [2,3]. Extrinsic causes of tracheobronchial obstruction include vascular anomalies such as innominate artery compression, a vascular ring, pulmonary artery sling, aortic arch anomaly and dilated aorta, and pulmonary artery. Patients with arch obstruction and intracardiac defects have a high probability of abnormal aortopulmonary geometry which causes airway compression. The tissue-to-tissue arch repair technique could result in severe airway complications such as compression of the left main bronchus and which was not a problem before the correction [1]. The association of cardiac defects and vascular tracheobronchial compression deserves special attention, as delayed diagnosis and treatment significantly increase the potential morbidity and mortality. Simultaneous repair of cardiac defects and vascular tracheobronchial compression syndromes also carries a higher risk of patient morbidity and mortality [4].

Cardiac defects likely to result in significant pulmonary artery enlargement include larger VSD, PDA, and atrioventricular canal. Tetralogy of Fallot (TOF which includes VSD) with an absent pulmonary valve, as seen in our case 2 , as a result of the close proximity of the pulmonary arteries to the bronchi and large right-to-left shunts, results in compression of the airways. The most frequent site of pulmonary-artery-induced bronchial compression is the superior aspect of the left main bronchus. The left main bronchus of the case 2 patient was severely compressed and this resulted in respiratory distress.

Tracheobronchial obstruction can vary in severity from mild, thus producing few or no symptoms, to severe and resulting in complete collapse of the trachea and recurrent apnea or spells. Infants' symptoms are more severe than those of adults due to the small size of the infants' airways and which thus limits any margin of safety. Infants and children with inspiratory and expiratory stridor and who have increased difficulty breathing exhibited by chest-wall retractions and prolonged expiration, should have prompt diagnostic evaluation of their airway. Methods for preoperative evaluation include $\mathrm{CT}$ and magnetic resonance (MR) imaging. CT and MR imaging have become the most useful modalities for assessing tracheal stenosis and various vascular malformations that either cause or are associated with tracheal stenosis. CT and MR imaging can produce high-quality, three-dimensional reconstruction of all of the anatomic elements, thus allowing precise anatomic delineation and improved surgical planning. However, CT and MR imaging are usually not available in the operating room. Therefore, we would emphasize the importance of an intraoperative bronchoscope used to guide the surgical maneuver and to prevent the need for additional surgery due to undiagnosed or newly developed airway collapse after corrective aortopexy.

Aortopexy, including pexis of the pulmonary artery trunk (PApexy), is accepted by clinicians as a successful surgical procedure for the treatment of severe tracheobronchial obstruction, including tracheobronchomalacia, and thus avoiding complex tracheobronchial resections. Early aortopexy can be recommended for infants with severe symptoms as well as short segment obstruction in the distal trachea causingsevere symptoms [5,6]. The infant-associated, complex intracardiac anomalies should undergo concomitant repair of both lesions of tracheobronchial compression and cardiac anomalies with the use of cardiopulmonary bypass. Sebening et al. [4] advocated early aggressive surgical intervention for both types of lesions so as to obtain better results in the management of infants and small children with this difficult and often fatal combination. Prolonged airway compression in the setting of congenital heart disease can result in frequent upper respiratory infections, wheezing, atelectasis, and pneumonia. And uncorrected extrinsic airway compression may result in tracheomalacia, bronchomalacia or both. Pulmonary-artery-induced bronchial compression has also been shown to be an important cause of congenital lobar emphysema [7].

Following aortopexy or PApexy, major postoperative issues are related to concurrent cardiac defects and residual airway disorders. Residual obstruction may be immediately observed on extubation or as a long-term problem after surgery. Roberts et al. [8] reported that difficulties of aortopexy for aortic arch anomalies were the result of airway compression. Pexis of the aorto-innominate artery can sometimes exaggerate the distortion of the lumen of the trachea, and thus requiring revision of the pexis [3].

The importance of bronchoscopy as both a diagnostic modality and a monitor of successful surgical manipulation is emphasized. As in case 3, failure to diagnose and/or to treat the intraoperative development of airway narrowing may result in postoperative respiratory embarrassment and failure to wean the patient from the mechanical ventilation. Since treatment of our case 3, our medical institution has routinely used bronchoscopy for the aortopexy in order to reduce the need for reoperation and to facilitate the intraoperative surgical decision-making even in those patients in whom airway obstruction did not exist preoperatively. We are concerned regarding the possibility of 
newly developed airway obstruction following a surgical procedure. Many published reports have demonstrated the usefulness of intraoperative bronchoscopic monitoring. These reports showed that intraoperative bronchoscopic results improved the chance for favorable clinical outcomes in patients with tracheobronchomalacia caused by a great vessel, vascular ring or innominate artery anomaly [2,9-11]. However, those reports are related to the corrective surgery performed for tracheobronchomalacia. Our case report is the only one which demonstrates the importance of intraoperative bronchoscopy for those patients who are undergoing corrective surgery for arch obstruction and intracardiac defects that cause abnormal aortopulmonary geometry. At our medical institution, intraoperative bronchoscopy is routinely used for airway evaluation during corrective surgery of the aorta or for pulmonary artery anomalies.

In conclusion, simultaneous repair of cardiac defects and vascular tracheobronchial obstruction has a high risk, however, uncorrected disease or surgical mismanagement may require repeat surgery and/or cause severe complications. Intraoperative bronchoscopy can provide guidance for the treatment of persistent narrowing of an airway or aggravation or new development of airway compression following surgical manipulation. It also allows the prevention of repeated surgery as well as postoperative complications. We recommend intraoperative bronchoscopic guidance in order to improve the postoperative outcomes of surgical procedures, including aortopexy and tracheobronchial decompression.

\section{References}

1. Jhang WK, Park JJ, Seo DM, Goo HW, Gwak M. Perioperative evaluation of airways in patients with arch obstruction and intracardiac defects. Ann Thorac Surg 2008, 85: 1753-8.

2. Filston HC, Ferguson TB Jr, Oldham HN. Airway obstruction by vascular anomalies. Importance of telescopic bronchoscopy. Ann Surg 1987; 205: 541-9.

3. DeLorimier AA, Harrison MR, Hardy K, Howell LJ, Adzick NS. Tracheobronchial obstructions in infants and children. Experience with 45 cases. Ann Surg 1990; 212: 277-89.

4. Sebening C, Jakob H, Tochtermann U, Lange R, Vahl CF, Bodegom P, et al. Vascular tracheobronchial compression syndromes-- experience in surgical treatment and literature review. Thorac Cardiovasc Surg 2000; 48: 164-74.

5. Abdel-Rahman U, Ahrens P, Fieguth HG, Kitz R, Heller K, Moritz A. Surgical treatment of tracheomalacia by bronchoscopic monitored aortopexy in infants and children. Ann Thorac Surg 2002; 74: 315-9.

6. Clevenger FW, Othersen HB Jr, Smith CD. Relief of tracheal compression by aortopexy. Ann Thorac Surg 1990; 50: 524-9: 524-8; discussion 529 .

7. Stanger P, Lucas RV Jr, Edwards JE. Anatomic factors causing respiratory distress in acyanotic congenital cardiac disease. Special reference to bronchial obstruction. Pediatrics 1969; 43: 760-9.

8. Roberts CS, Othersen HB Jr, Sade RM, Smith CD 3rd, Tagge EP, Crawford FA Jr. Tracheoesophageal compression from aortic arch anomalies: analysis of 30 operatively treated children. J Pediatr Surg 1994; 29: 334-7; discussion 337-8.

9. Kamata S, Usui N, Sawai T, Nose K, Kitayama Y, Okuyama H, et al. Pexis of the great vessels for patients with tracheobronchomalacia in infancy. J Pediatr Surg 2000; 35: 454-7.

10. Weber TR, Keller MS, Fiore A. Aortic suspension (aortopexy) for severe tracheomalacia in infants and children. Am J Surg 2002; 184: 573-7; discussion 577.

11. Hagl S, Jacob H, Sebening C, van Bodegrom P, Schmidt K, Zilow E, et al. External stabilization of long-segment tracheobronchomalacia guided by intraoperative bronchoscopy. Ann Thorac Surg 1997; 64: 1412-20; discussion 1421. 